\title{
A comparison of fulvestrant plus a targeted agent with fulvestrant alone in hormone receptor- positive advanced breast cancer that progressed on prior endocrine therapy: a meta-analysis
}

This article was published in the following Dove Press journal:

OncoTargets and Therapy

\section{Liang Xu, ${ }^{1, *}$ Ningning Yan, ${ }^{2, *}$ Zhihua Li,' Lihua Luo, ${ }^{3}$ Xiaobo Wu,' Qiuming Liu,' Yingchun $\mathrm{Xu},{ }^{4}$ Yali $\mathrm{Cao}{ }^{1}$ \\ 'The First Department of Prevention and Cure Centre of Breast Disease, The Third Hospital of Nanchang City, Key Laboratory of Breast Disease in Jiangxi Province, Nanchang, JiangXi 330009, China; ${ }^{2}$ Department of Oncology, Shanghai Ruijin Hospital, Shanghai Jiaotong University School of Medicine, Shanghai 200025, China; ${ }^{3}$ Medical Department, Graduate School of Nanchang University, Nanchang, Jiangxi 330006, China; ${ }^{4}$ Department of Oncology, Shanghai Renji Hospital, Shanghai Jiaotong University School of Medicine, Shanghai 200I27, China \\ *These authors contributed equally to this work}

\section{Correspondence: Yingchun Xu}

Department of Oncology, Shanghai Renji Hospital, Shanghai Jiaotong University

School of Medicine, No. I27, Pujian Road,

Pudong District, Shanghai 200127, China

Tel +862 2। 6838313 |

Fax +862158394262

Email xiaoxu2384@I63.com

Yali Cao

The First Department of Prevention and Cure Center of Breast Disease, The Third Hospital of Nanchang City, Key Laboratory of Breast Disease in Jiangxi Province, Nanchang, JiangXi 330009, China

Tel +8679186646463

Email caoyali@medmail.com.cn

\begin{abstract}
Fulvestrant is recommended for the hormone receptor-positive metastatic breast cancer (MBC) patients progressed during or after prior endocrine therapy. Notably, recent evidence has also demonstrated that adding a targeted agent to fulvestrant conferred a significantly clinical benefit in these patients. Since these results were inconsistent among the studies, this meta-analysis herein was conducted to compare the efficacy and toxicities of the fulvestrant-based combination therapy with fulvestrant monotherapy. Thus, a systemic research was performed in PubMed, Embase, and Cochrane library to identify relevant Phase II or Phase III randomized controlled trials. The progression-free survival (PFS), overall response rate (ORR), and toxicities were evaluated. And HR, risk ratio (RR), and their 95\% CIs were employed to complete the pooled analyses. In total, 13 studies with 3,910-hour positive MBC patients progressed on prior endocrine therapy were included in our meta-analysis. Improvements of doublet-agents group were proven in terms of PFS (HR 0.73, 95\% CI $=0.63-0.86, P=0.000)$ and ORR (RR $2.07,95 \% \mathrm{CI}=1.67-2.58, P=0.000)$. And the further subgroup analysis also demonstrated that fulvestrant in combination with a cyclin-dependent kinase (CDK4/6) inhibitor or a PI3K/mTOR inhibitor was associated with a superior efficacy (RR 2.72, 95\% CI $=1.93-3.83, P=0.000$ and RR $1.60,95 \% \mathrm{CI}=1.15-2.23, P=0.005$, respectively). However, the efficacy was comparable between the other combination strategies and fulvestrant alone. With respect to the adverse effects, adding a targeted agent to fulvestrant also produced more frequent grade $3 / 4$ toxicities (RR 3.86, 95\% CI $=2.66-5.61, P=0.000$ ). Taken together, combination of fulvestrant with a targeted agent, especially inhibitors targeting CDK $4 / 6$ or PI3K/mTOR pathway, may open a new avenue for more effective therapies in relapse or metastatic hormone receptor-positive breast cancer after prior aromatase inhibitors or tamoxifen treatment. In addition, identifying reliable biomarkers to delineate which subgroup of patients will specially benefit from fulvestrant-based combination therapy is warranted.
\end{abstract}

Keywords: fulvestrant, breast cancer, combination therapy, endocrine resistance

\section{Introduction}

Breast cancer is the most prevalent malignancy worldwide with almost 268,670 cases newly diagnosed in 2018 in the United States only. ${ }^{1}$ Although major progress has been made in cancer treatment, a substantial proportion of the breast cancer patients still experience disease progression, relapse, and reduced overall survival (OS), ${ }^{2}$ and $\sim 70 \%$ of them are hormone receptor-positive. ${ }^{3}$ Currently, for hormone receptor-positive advanced breast cancer without visceral crisis, endocrine therapy is regarded as the 
cornerstone treatment because of the decent disease control and improved quality of life. ${ }^{4}$ However, virtually all the patients will develop disease progression either as de novo resistance or as acquired resistance to the endocrine therapy. ${ }^{5}$ And the management of the endocrine resistance is still a challenging scenario with many unanswered questions.

Fulvestrant, a high selective estrogen receptor downregulator, is able to bind, block, and accelerate the estrogen receptor degradation. ${ }^{6}$ In the postmenopausal hormone receptor-positive and human epidermal growth factor receptor 2 (HER2)-negative advanced breast patients, recent evidence have validated the role of Fulvestrant monotherapy both in the completely endocrine-naive patients ${ }^{7,8}$ and in the patients progressed while receiving or shortly after the completion of endocrine therapy. ${ }^{9,10}$ Currently, fulvestrant is approved for the treatment of postmenopausal women with advanced breast cancer after recurrence or first progression following anti-estrogen therapy. ${ }^{11}$

Nevertheless, the landscape of the treatment of hormone receptor-positive advanced breast cancer is changing rapidly. With increasing understanding of the underlying biological mechanisms of endocrine resistance for metastatic breast cancer (MBC), various clinical trials evaluating fulvestrant in combination with a targeted agent such as cyclin-dependent kinase 4/6 (CDK4/6) inhibitor and PI3K/mTOR pathway inhibitor have been conducted. ${ }^{12,13}$ Several fulvestrant-based combination strategies were found to associate with a significant prolonged progression-free survival (PFS) and manageable toxicities in hormone receptor-positive patients with metastatic disease who progressed after a prior endocrine therapy when compared with the fulvestrant alone. ${ }^{12-14}$ However, some other trials failed to demonstrate a better efficacy of the doublet agents. ${ }^{15-17}$ Given these inconsistent results, selecting the optimal choice for these patients becomes complex and somewhat a challenge to the treating clinicians. And the hardest question is whether to choose fulvestrant singleagent therapy or fulvestrant combination therapy, because both of them seem to be reasonable alternatives. ${ }^{4}$

Herein, our meta-analysis was performed to evaluate the efficacy and safety of fulvestrant combined with a targeted agent compared with fulvestrant monotherapy in those hormone receptor-positive $\mathrm{MBC}$ patients progressed on prior endocrine therapy.

\section{Methods}

\section{Searching strategy and inclusion criteria}

A literature search was conducted to identify Phase II or Phase III randomized controlled trials (RCTs), which compared fulvestrant single agent with fulvestrant plus targeted agents in hormone receptor-positive MBC progressed on previous endocrine therapy from January 1, 1990 to December 10, 2017 from the PubMed, Cochrane library, and Embase. The ClinicalTrials.gov network (https://ClinicalTrials.gov) was also searched for the ongoing studies. In addition, posters and abstracts of the annual meeting of European Society of Medical Oncology and San Antonio Breast Cancer Symposium in the past 10 years were also scanned. The search algorithm was (breast OR mammary) AND (carcinoma OR neoplasm OR tumor OR cancer) AND (metastatic OR advanced OR relapse*) AND (pretreat*) AND (fulvestrant). All the reference list of the original papers was reviewed as well.

The inclusion criteria were: (1) prospective Phase II or Phase III RCTs. (2) RCTs comparing fulvestrant monotherapy with the fulvestrant plus a targeted agent in hormone receptor-positive $\mathrm{MBC}$ progressing after endocrine therapy. (3) The studies must have sufficient data for extraction, stratification, and calculation.

\section{Quality assessment of the studies}

Two investigators carefully evaluated the quality and the eligibility of the studies independently. Disagreements were resolved after discussion with the third investigator. Using the methods previously reported by Jadad et al, ${ }^{18}$ we assessed quality of the trials according to the following three questions: (1) whether the trial reported an appropriate randomization method ( $0-2$ scores); (2) whether the report included an appropriate blinding method (0-2 scores); and (3) whether the report included an account of the number of withdrawals or dropouts.

\section{Data extraction}

All the data extracted were filled into a standard form by two independent investigators. Discrepancies were resolved through discussion. The following information were extracted: (1) general information of each study, such as journal name, authors' names, and date of publication; (2) characteristics of the patients such as age, sex, race, menopause, HR status, performance status, and tumor burden; (3) study design, such as samples of each group, randomization and blinding method, inclusion and exclusion criteria, type of study endpoints, and follow-up; and (4) data, such as regimens, drug dose, PFS, OS, overall response rate (ORR), clinical benefit rate (CBR), line of therapy, and the pathways inhibited.

\section{Statistical analysis}

Briefly, pooled HR and 95\% CI were employed to appraise the PFS. And pooled risk ratio (RR) and 95\% CI were used 
to evaluate the ORR, CBR, and adverse effects (AEs). HRs, RRs, and 95\% CIs were extracted from the original publications directly if they were reported. Otherwise, the original Kaplan-Meier curves of the included studies were read, and the HRs, RRs, and 95\% CIs were calculated using the Engauge Digitizer software as previously reported. ${ }^{19}$ This is a free software that can be downloaded from $\underline{h t t p: / / s o u r c e-}$ forge.net/projects/digitizer/. In addition, $\chi^{2}$-based $Q$-test was used to estimate the heterogeneity between the groups. ${ }^{20}$ Heterogeneity was considered exist when the $P_{\text {heterogeneity }}<0.1$ or $I^{2}>50 \%$, and a random effect model was used. Otherwise, a fixed effect model was used. ${ }^{21,22}$ Moreover, publication bias was evaluated using the Egger's test and Begg's test. All the calculation and estimation was conducted using the STATA version 12.0 software (StataCorp LP, College Station, TX, USA). Of note, HR $>1$ suggests more PFS events in the combination group compared with single-agent group; $\mathrm{RR}<1$ suggests more relevant AEs and better treatment response in the combination group, and vice versa.

\section{Results}

\section{Study search and eligibility}

As shown in Figure 1, a total of 1,072 records were identified using our aforementioned searching strategy, and three more studies were added manually from other sources including reference list of original articles, international meeting reports, and reviews. Subsequently, 184 duplicates were removed. And we screened the titles and abstracts of the remaining 891 records, then, 843 were excluded because they were irrelevant publications or reviews. At last, of the 48 potential studies, only 13 studies $^{12-17,23-29}$ were included in our qualitative synthesis based on our inclusion criteria.

\section{Study characteristics and patients}

In total, 3,910-hour positive MBC patients progressed on prior endocrine therapy were included in our meta-analysis. Of them, 2,247 patients were randomized to the fulvestrant plus a targeted-agent group and 1,663 patients were randomized to fulvestrant single-agent group. The characteristics of the studies were listed in Table 1 . In the 13 eligible studies, 8 were Phase II and 5 were Phase III RCTs. The standard dose of fulvestrant (500 mg monthly) was administered in 11 studies, and the other 2 included studies ${ }^{16,24}$ utilized a suboptimal dose of fulvestrant at $250 \mathrm{mg}$ monthly. As shown in Table 2, not all patients were HER2 negative and postmenopausal. In detail, $68(3 \%)$ patients were HER2 positive and $224(10 \%)$ patients were premenopausal or perimenopausal, respectively. And it should be noted that all the premenopausal or perimenopausal patients were concurrently given a luteinizing-hormone-releasing-hormone agonist (LHRHa) before the fulvestrant monotherapy or combination therapy.

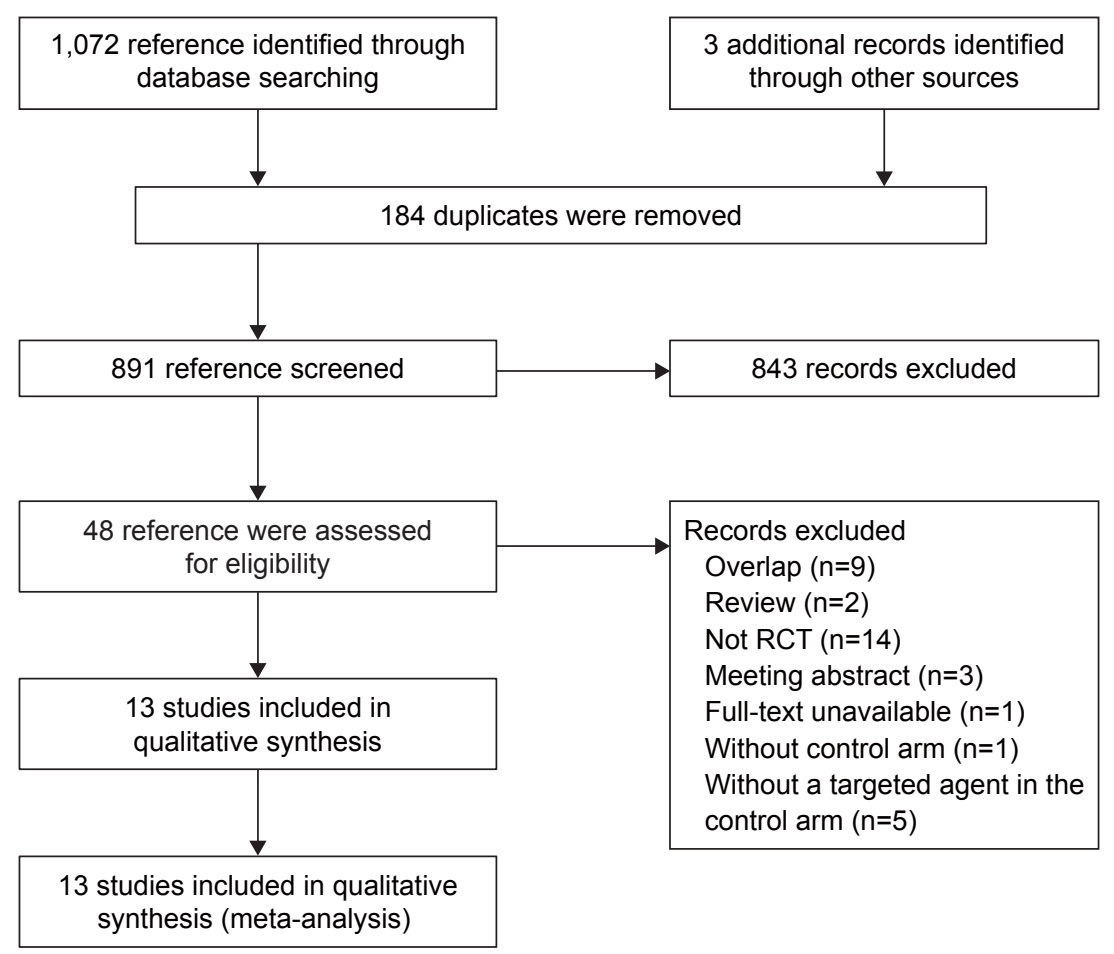

Figure I Flow diagram and results of our literature identification.

Abbreviation: RCT, randomized controlled trial. 
Table I Characteristics of 13 trials eligible for meta-analysis

\begin{tabular}{|c|c|c|c|c|c|c|c|}
\hline Author & Year & Phase & Regimens & $\begin{array}{l}\text { Dose } \\
\text { of FUL }\end{array}$ & $\begin{array}{l}\text { Pathways } \\
\text { inhibited }\end{array}$ & $\begin{array}{l}\text { Targeted } \\
\text { agents }\end{array}$ & $\begin{array}{l}\text { Jadac } \\
\text { score }\end{array}$ \\
\hline Sledge GW'14 & 2017 & III & FUL + abemaciclib/FUL & $500 \mathrm{mg}$ & CDK4/6 & Abemaciclib & 3 \\
\hline Zaman $\mathrm{K}^{15}$ & 2015 & II & FUL + selumetinib/FUL & $500 \mathrm{mg}$ & MEKI/2 & Selumetinib & 3 \\
\hline Adelson $\mathrm{K}^{23}$ & 2016 & II & FUL + bortezomib/FUL & $500 \mathrm{mg}$ & Proteasome & Bortezomib & 3 \\
\hline Cristofanilli $M^{12}$ & 2016 & III & FUL + palbociclib/FUL & $500 \mathrm{mg}$ & CDK4/6 & Palbociclib & 3 \\
\hline Hyams DM ${ }^{24}$ & 2013 & II & FUL + cediranib/FUL & $250 \mathrm{mg}$ & VEGF & Cediranib & 3 \\
\hline Burstein $\mathrm{HJ}^{16}$ & 2014 & III & FUL + lapatinib/FUL & $250 \mathrm{mg}$ & HERI, HER2 & Lapatinib & 3 \\
\hline Clemons $\mathrm{MJ}^{17}$ & 2014 & II & FUL + vande/FUL & $500 \mathrm{mg}$ & VEGF, EGFR & Vandetanib & 3 \\
\hline Musolino $\mathrm{A}^{25}$ & 2017 & II & FUL + dovitinib/FUL & $500 \mathrm{mg}$ & FGFR & Dovitinib & 3 \\
\hline Baselga J'3 & 2017 & III & FUL + buparlisib/FUL & $500 \mathrm{mg}$ & $\mathrm{PI} 3 \mathrm{~K}$ & Buparlisib & 3 \\
\hline Kornblum NS ${ }^{26}$ & 2016 & II & FUL + everolimus/FUL & $500 \mathrm{mg}$ & mTOR & Everolimus & 3 \\
\hline Krop IE ${ }^{27}$ & 2016 & II & FUL + pictilisib/FUL & $500 \mathrm{mg}$ & $\mathrm{PI} 3 \mathrm{~K}$ & Pictilisib & 3 \\
\hline Di Leo $A^{28}$ & 2017 & III & FUL + buparlisib/FUL & $500 \mathrm{mg}$ & $\mathrm{PI} 3 \mathrm{~K}$ & Buparlisib & 3 \\
\hline Robertson $\mathrm{JF}^{29}$ & 2013 & ॥ & FUL + ganitinib/FUL & $500 \mathrm{mg}$ & IGF-I/IGF-2 & Ganitumab & 3 \\
\hline
\end{tabular}

Abbreviations: CDK4/6, cyclin-dependent kinase 4/6; FUL, fulvestrant; HERI, human epidermal growth factor receptor I; HER2, human epidermal growth factor receptor 2.

\section{Progression-free survival}

In this meta-analysis, the HRs and 95\% CIs for PFS were given directly in 12 original papers included, and the related data in one study were unavailable. ${ }^{15}$ We used the random model to evaluate the pooled PFS because the obvious heterogeneity existed $\left(I^{2}=72.7 \%\right)$. Our results demonstrated that the addition of a targeted agent to fulvestrant was associated with a prolonged PFS when compared with fulvestrant alone (HR 0.73, 95\% CI $=0.63-0.86, P=0.000$, Figure 2 ). In our further subgroup analyses, combination therapy including targeted-agent blocking of CDK $4 / 6$ or PI3K/mTOR-signaling pathway contributed to a significantly improved PFS. However, in the VEGF or other inhibitors containing combination regimen, the clinical benefits of the doublet agents were similar to that of fulvestrant alone (CDK4/6 inhibitors: HR $0.51,95 \% \mathrm{CI}=0.43-0.61, P=0.000$; VEGF inhibitors, HR $0.92,95 \% \mathrm{CI}=0.66-1.28, P=0.623$; PI3K/mTOR inhibitors: HR 0.73, 95\% CI $=0.66-0.81, P=0.000$; others: HR 0.93, $95 \% \mathrm{CI}=0.75-1.15, P=0.502$, Figure 2 ).

\section{Overall response rate}

The ORR data were unavailable in three studies. ${ }^{23,26,29}$ Since no significant heterogeneity found $\left(I^{2}=30.5 \%\right)$, the pooled RR was calculated using fixed model subsequently. Similar to the PFS, the combination therapy also improved ORR compared with fulvestrant alone in the hormone receptor-positive MBC patients progressed on prior endocrine therapy (RR $2.07,95 \% \mathrm{CI}=1.67-2.58, P=0.000$, Figure 3 ). In parallel,

Table 2 Patients characteristics and outcomes in this meta-analysis

\begin{tabular}{|c|c|c|c|c|c|c|}
\hline Author & $\begin{array}{l}\text { No of } \\
\text { patients }\end{array}$ & $\begin{array}{l}\text { Postmenopausal } \\
\text { status (\%) }\end{array}$ & $\begin{array}{l}\text { HER2 } \\
\text { status }\end{array}$ & $\begin{array}{l}\text { ORR (doublet } \\
\text { agents vs single } \\
\text { agent) }\end{array}$ & $\begin{array}{l}\text { Median PFS } \\
\text { (months, } \\
\text { doublet agents } \\
\text { vs single agent) }\end{array}$ & $\begin{array}{l}\text { Median OS } \\
\text { (months, } \\
\text { doublet agents } \\
\text { vs single agent) }\end{array}$ \\
\hline Sledge $\mathrm{GW}^{14}$ & 669 & 83 & - & $35 \%$ vs $16 \%$ & I6.4 vs 9.3 & - \\
\hline Zaman $\mathrm{K}^{15}$ & 42 & 100 & - & $5 \%$ vs $15 \%$ & 3.7 vs 5.6 & 22.9 vs 19.4 \\
\hline Adelson $\mathrm{K}^{23}$ & 116 & 100 & - & - & 2.73 vs 2.69 & - \\
\hline Cristofanilli $M^{\prime 2}$ & 521 & 79 & - & $19 \%$ vs $9 \%$ & 9.5 vs 4.6 & - \\
\hline Hyams DM ${ }^{24}$ & 62 & 100 & NA & $22 \%$ vs $8 \%$ & 7.4 vs 3.7 & - \\
\hline Burstein $\mathrm{HJ}^{16}$ & 291 & 100 & $\pm 18 \%$ & $20 \%$ vs $9 \%$ & 4.7 vs 3.8 & 30 vs 26.4 \\
\hline Clemons $\mathrm{MJ}{ }^{17}$ & 129 & 100 & $\pm 5 \%$ & $0 \%$ vs $7 \%$ & 5.8 vs 4.8 & 31 vs - \\
\hline Musolino $\mathrm{A}^{25}$ & 97 & 100 & - & $28 \%$ vs $10 \%$ & 5.5 vs 5.5 & - \\
\hline Baselga J'3 & $\mathrm{I}, \mathrm{I} 47$ & 100 & - & $12 \%$ vs $8 \%$ & 6.9 vs 5.0 & - \\
\hline Kornblum NS ${ }^{26}$ & 130 & 100 & - & - & I0.4 vs 5.1 & - \\
\hline Krop IE ${ }^{27}$ & 168 & 100 & - & $8 \%$ vs $6 \%$ & 6.6 vs 5.1 & - \\
\hline Di Leo $A^{28}$ & 432 & 100 & - & $8 \%$ vs $2 \%$ & 3.9 vs 1.8 & - \\
\hline Robertson JF ${ }^{29}$ & 156 & 100 & $\pm 7 \%$ & - & 3.7 vs 5.4 & - \\
\hline
\end{tabular}

Abbreviations: HER2, human epidermal growth factor receptor 2; ORR, overall response rate; OS, overall survival; PFS, progression-free survival; NA, not available. 
PFS

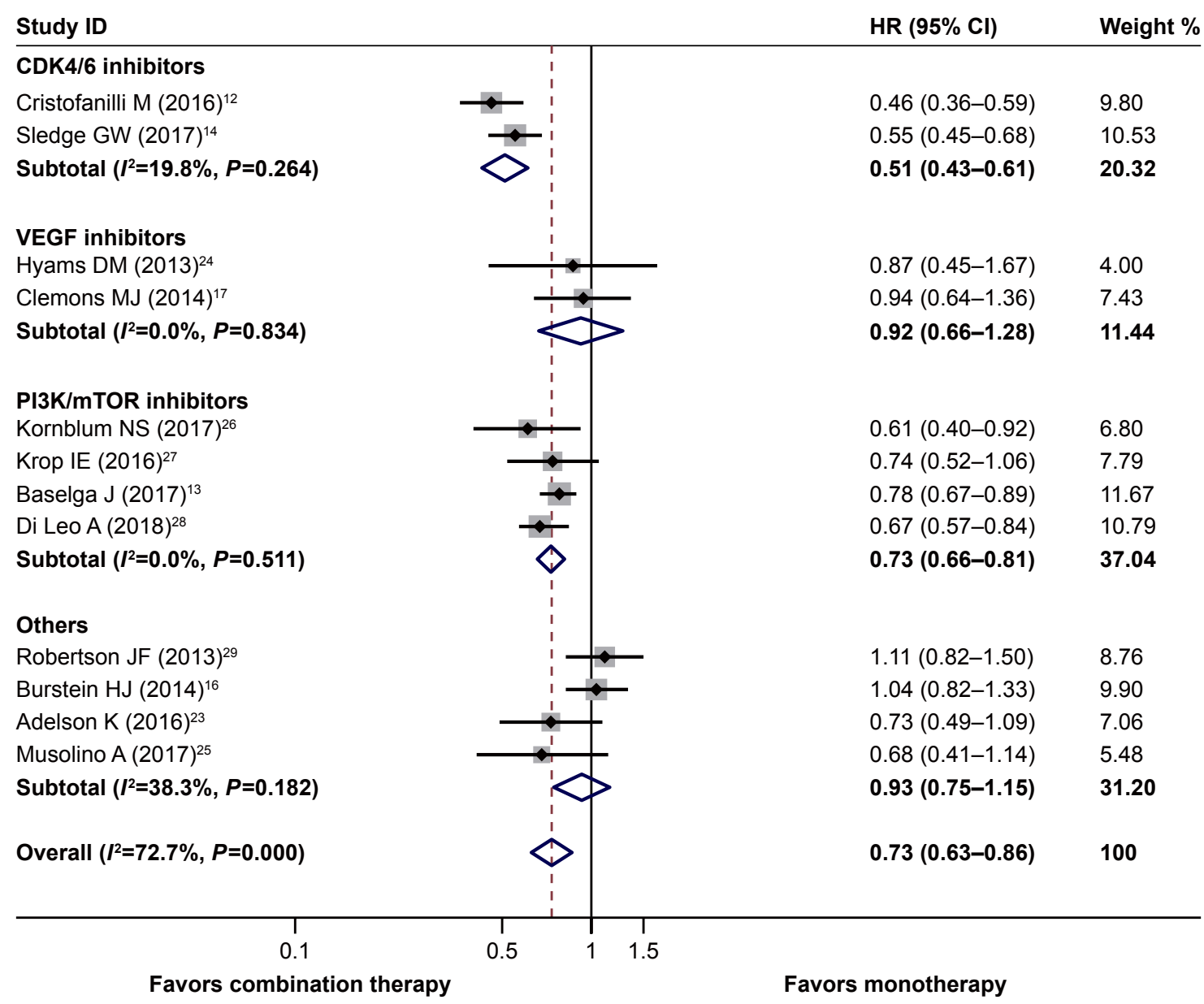

Figure 2 The pooled HR and $95 \% \mathrm{Cl}$ for PFS following fulvestrant in combination with a targeted agent and fulvestrant alone. Note: Weights are from random effect analysis.

Abbreviations: PFS, progression-free survival; CDK4/6, cyclin-dependent kinase 4/6.

the subgroup analyses also revealed that fulvestrant plus CDK4/6 inhibitors or PI3K/mTOR inhibitors significantly increased the ORR compared with fulvestrant monotherapy (RR 2.72, 95\% CI $=1.93-3.83, P=0.000$ and RR $1.60,95 \%$ $\mathrm{CI}=1.15-2.23, P=0.005$, respectively, Figure 3 ).

\section{Toxicities and safety}

Concerning the toxicities and safety, more side effects and drug-related treatment withdrawals were observed in the combination group in the majority of the included studies. Ten studies reported the grade 3/4 toxicities,,$^{12,14-17,24-28}$ and the pooled analysis showed that the doublet-agent group produced more toxicities although most of them were manageable (RR 3.86, 95\% CI =2.66-5.61, $P=0.000$, Figure 4). In addition, in the subgroup analyses, those inhibitors targeting CDK4/6, VEGF, or PI3K/mTOR pathway plus fulvestrant led to a higher incidence of grade $3 / 4$ toxicities. And no substantial difference in the grade $3 / 4$ toxicities was observed in other agents combination group compared with fulvestrant alone (CDK4/6 inhibitors: HR 6.90, 95\% $\mathrm{CI}=3.84-12.40, P=0.000$; VEGF inhibitors: HR 2.80, 95\% CI $=1.27-6.20, P=0.011$; PI3K/mTOR inhibitors: HR $3.44,95 \% \mathrm{CI}=2.50-4.74, P=0.000$; others: HR $2.49,95 \%$ $\mathrm{CI}=0.78-7.93, P=0.122$, Figure 4$)$.

\section{Publication bias}

In order to minimize the potential publication bias and to acquire a reliable result, several steps were taken subsequently. First, our literature search strategy was extensive and accurate. Second, we selected the included studies strictly according to our inclusion and exclusion criteria. Finally, we also analyzed the publication bias by several methods. And as is shown in Figure 5A and B, no significant publication bias was observed in our meta-analysis (PFS: Begg's test: $P=0.945$, Egger's test: $P=0.782$; ORR: Begg's test: $P=0.721$, Egger's test: $P=0.681$ ). 


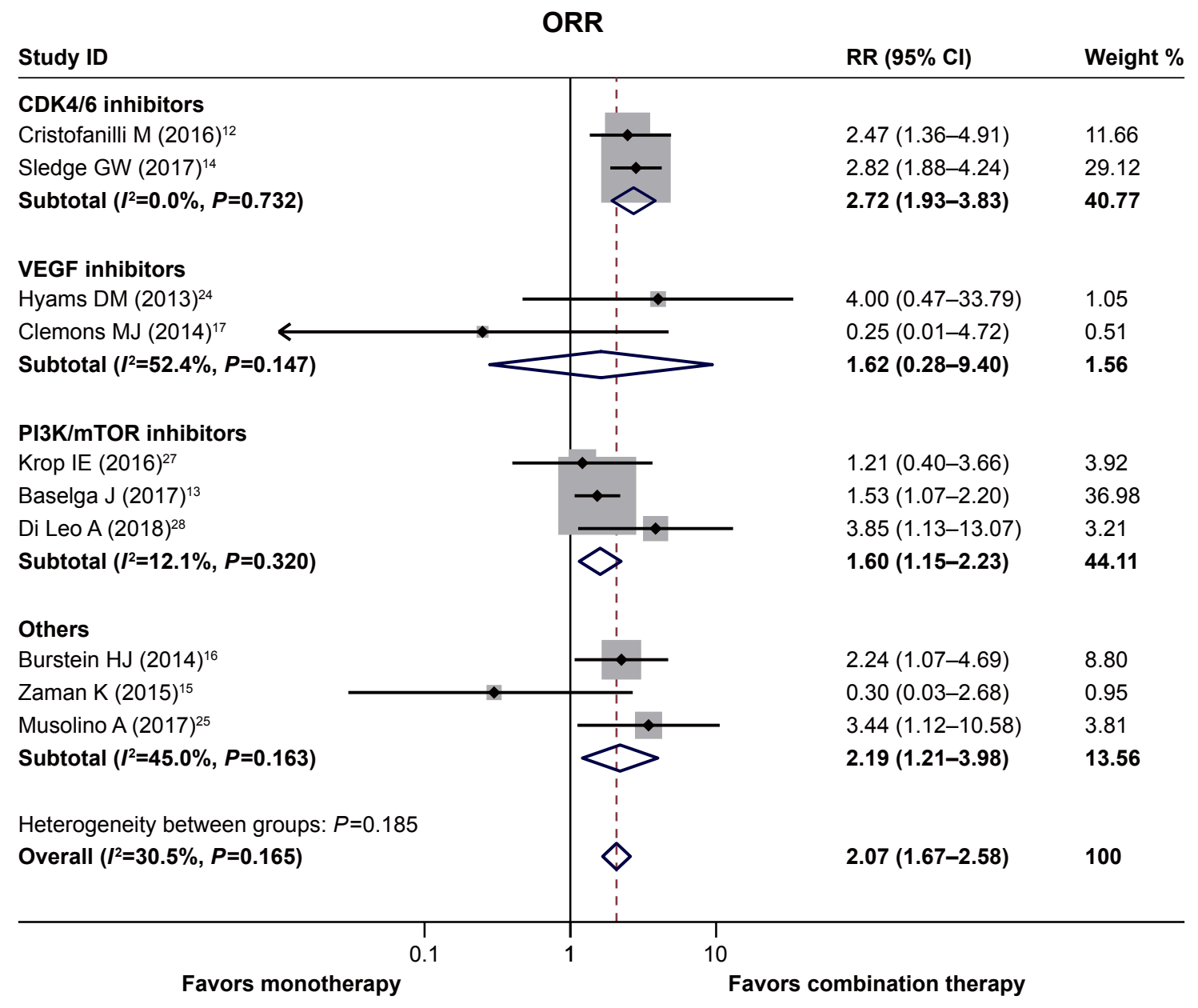

Figure 3 The pooled risk ratio (RR) and $95 \% \mathrm{Cl}$ for overall response rate. Abbreviations: ORR, overall response rate; CDK4/6, cyclin-dependent kinase 4/6.

\section{Discussion}

Currently, the optimal treatment for the hormone receptorpositive MBC patients progressed on previous endocrine therapy has not been well-defined. ${ }^{4}$ First, fulvestrant was approved for the treatment of postmenopausal advanced breast cancer patients after recurrence or first progression following endocrine therapy. ${ }^{30,31}$ However, with the landscape of hormone therapy for this population rapidly reshaping, fulvestrant was found to be a good platform for combining agents targeting other pathways involved in the anti-estrogen therapy resistance. ${ }^{23}$ In the PALOMA-3 trial, fulvestrant plus palbociclib (a CDK4/6 inhibitor) significantly extended the PFS compared with fulvestrant single agent irrespective of the prior endocrine sensitivity, HR expression level, and PI3KCA mutation status. ${ }^{12}$ And in the BELLE-2 trial, fulvestrant in combination with buparlisib, an oral pan-PI3K inhibitor, also resulted in a superior efficacy compared with fulvestrant alone. ${ }^{13}$ Notably, Lin et al had performed a meta-analysis and compared the efficacy and toxicities of fulvestrant plus targeted agents and fulvestrant alone in this population before. ${ }^{32}$ Unfortunately, only eight studies included and no subgroup analyses performed made the results somewhat problematic and unstable. More importantly, several related trials evaluating the fulvestrantbased combination therapy were reported recently, ${ }^{13,14,26,28}$ and they will definitely affect the pooled results eventually. Therefore, we re-searched the related RCTs and updated the meta-analysis accordingly in order to solve this burning clinical problem.

In this meta-analysis, our results indicated that fulvestrant in combination with a targeted agent appeared to be more efficacious than fulvestrant alone in terms of PFS and ORR. And these superiority were further confirmed in the combination strategies of fulvestrant with CDK4/6 inhibitors or PI3K/mTOR pathway inhibitors in the subgroup analyses (Figures 2 and 3). These findings taken together suggested 


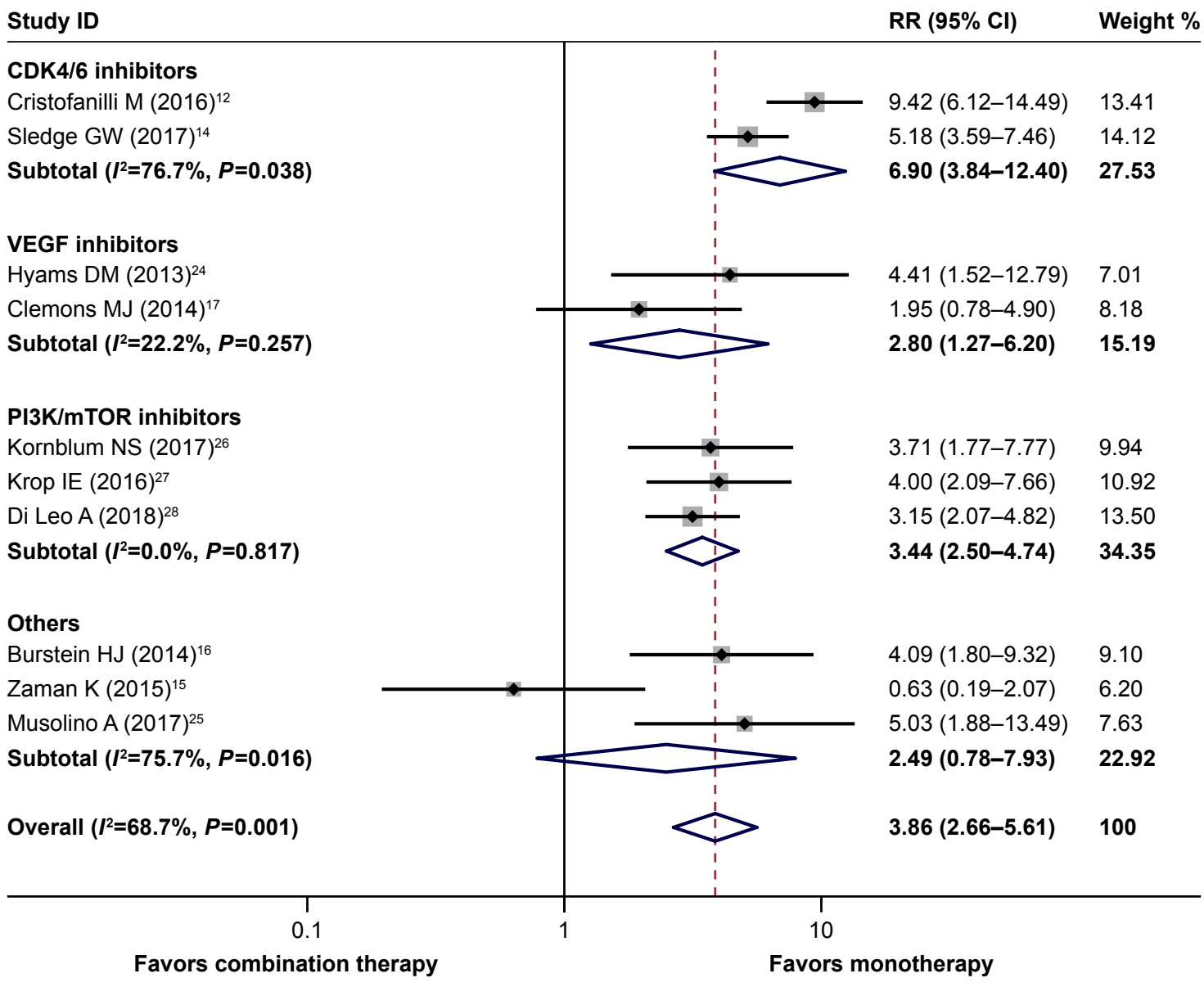

Figure 4 Forest plot of relative risk of treatment induced grade 3/4 toxicities. Note: Weights are from random effects analysis.

Abbreviations: RR, risk ratio; CDK4/6, cyclin-dependent kinase 4/6.

that the superior clinical efficacy of doublet agents might depend on the specific therapeutic combinations. Hence, for the hormone receptor-positive $\mathrm{MBC}$ patients, relapse after endocrine therapy, fulvestrant in combination with agents targeting CDK4/6 or PI3K/mTOR represented a promising approach to overcome or at least delay the endocrine resistance. However, although PI3K mutation is already recognized as a predictor of response to anti-PI3K targeted therapy in breast cancer in several trials, the majority of our eligible trials in this meta-analysis did not report the PI3K mutation data directly. Therefore, clinical trials aimed to determine the benefits of fulvestrant in combination with PI3K inhibitor in the PI3K-pathway activated subgroup is warranted in future.

With respect to the toxicities, we also found the grade $3 / 4$ AEs were more common in the combination group irrespective of the targeted agents included (Figure 4). Considering the first priority of $\mathrm{MBC}$ treatment is to improve or at least maintain the quality of life and to prolong survival, ${ }^{4}$ thus we should be vigilant about the uncontrolled side effects outweighing the marginal clinical benefits. With regret, identification of the most suitable hormone receptor-positive MBC patients who can benefit most from the fulvestrantbased combination therapy is still a significant challenge. The good news is that the circulating tumor DNA (ctDNA), which is released from apoptotic or necrotic tumor cells, seems to be a promising candidate because it reflects the mutation status after relapse. ${ }^{33}$ Moreover, the ctDNA analysis enable us to evaluate the mutational and expression changes which reflect the patient-specific dynamic tumor landscapes in a repetitive and non-invasive way. ${ }^{34}$ And our findings merit further investigation into the relationship between the ctDNA mutation status and the clinical benefit of the combination therapy in these patients. 


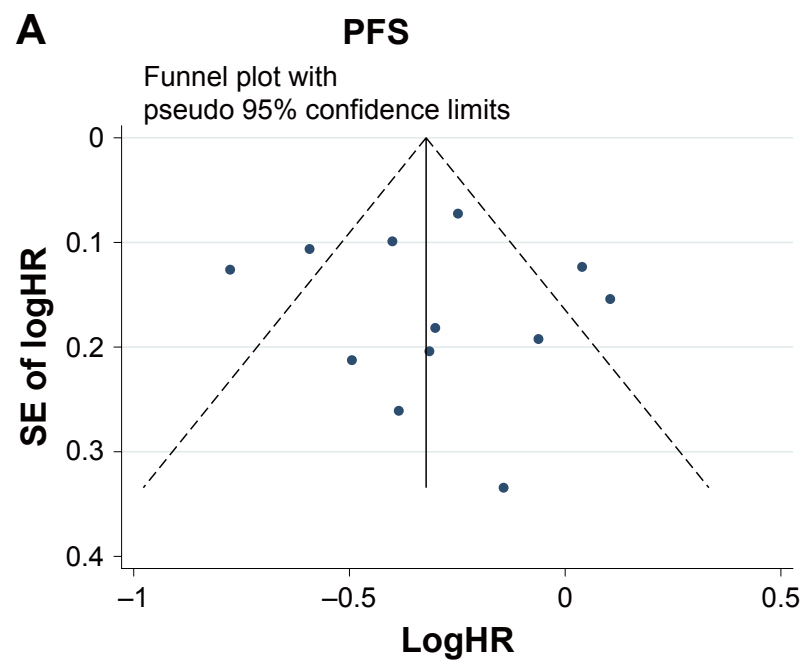

Figure 5 Funnel plot of publication bias in the meta-analysis. Notes: (A) PFS. (B) ORR.

Abbreviations: PFS, progression-free survival; ORR, overall response rate.

Honestly, there are several limitations in the present metaanalysis, and it should be cautious when interpreting the results. First, different combination strategies, treatment regimens, drug doses, and patient selections inevitably imposed heterogeneity to the pooled analyses. For instance, not all patients included in the meta-analysis were HER 2 negative (3\% HER2 positive) and postmenopausal (10\% perimenopausal or premenopausal). Since HER2 pathway is able to crosstalk with the estrogen-signaling pathway and is involved in the endocrine resistance, ${ }^{35,36}$ the HER2-positive population and anti-HER2 therapy themselves will definitely act as relative confounding factors in turn. Moreover, for the perimenopausal and premenopausal patients, we cannot rule out the possibility that the administered LHRHa may affect the final estimation. After all, LHRHa itself was reported as an effective endocrine approach in breast cancer. ${ }^{37,38}$ Second, the line of therapy, metastatic sites, tumor burden, prior anti-estrogen drugs, and anti-estrogen treatment sequence in these eligible studies were also heterogeneous. And the sensitivities to previous endocrine therapy were also not homogeneous. Notably, compared with hormone receptor-positive MBC patients with visceral metastases, fulvestrant was believed to be more efficacious for those patients with nonvisceral metastases only. ${ }^{7}$ Thus, the different metastatic sites and tumor burden of patients in these included studies may also contribute to the observed heterogeneity. Third, not all the included studies administered a standard dose of fulvestrant (500 mg monthly) to the patients. Fulvestrant is a pure, steroidal ER antagonist, and the inhibitory effect of the estrogen-signaling pathway is dose dependent. And the CONFIRM trial has revealed a superior effect of $500 \mathrm{mg}$ fulvestrant when compared with $250 \mathrm{mg}$ fulvestrant in terms

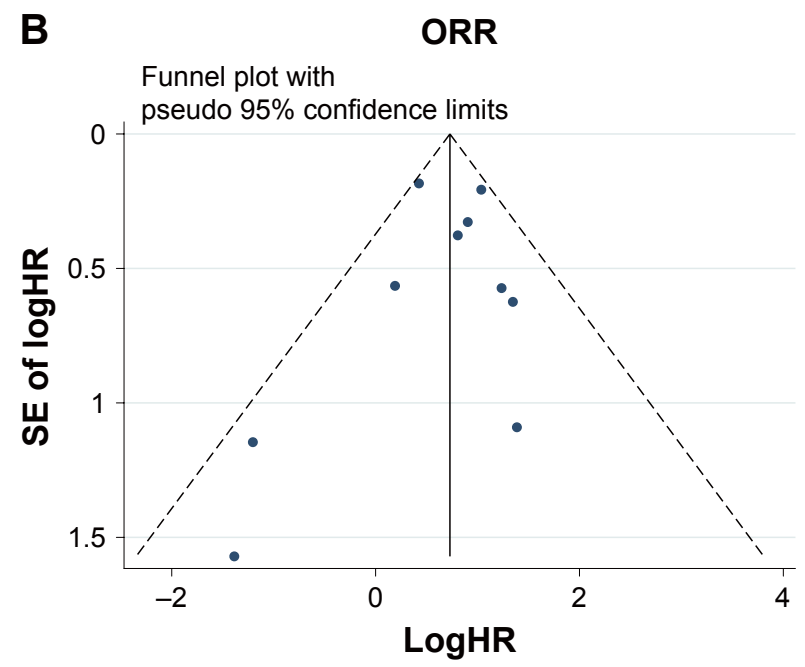

of PFS and OS. ${ }^{39}$ Therefore, the nonstandard fulvestrant dose may partly result in bias of our findings as well. Finally, the present meta-analysis was not conducted based on the individual patient data but on the HR and 95\% CIs of each study extracted. And the sample size was relatively limited, which made it incapable of comparing some kinds of infrequent toxicities between those groups. Despite all these limitations, our study is a meaningful meta-analysis to evaluate the fulvestrant plus a targeted agent in the hormone receptor-positive MBC progressed on previous endocrine therapy.

In conclusion, we validated the combination of a targeted agent with fulvestrant was associated with a superior benefit and more frequent AEs when compared with fulvestrant alone. This warrants that further studies focus on identification of those patients who will derive benefits from the combination strategy to aid treatment decisions.

\section{Acknowledgment}

This work was supported by the Youth Science Foundation of Jiangxi Province (No 20171BAB215045).

\section{Disclosure}

The authors report no conflicts of interest in this work.

\section{References}

1. Siegel RL, Miller KD, Jemal A. Cancer statistics, 2018. CA: Cancer J Clin. 2018;68(1):7-30.

2. Başaran GA, Twelves C, Diéras V, Cortés J, Awada A. Ongoing unmet needs in treating estrogen receptor-positive/HER2-negative metastatic breast cancer. Cancer Treat Rev. 2018;63:144-155.

3. Vidula N, Rugo HS. Emerging data on improving response to hormone therapy: the role of novel targeted agents. Expert Rev Anticancer Ther. 2018;18(1):3-18. 
4. Boér K. Fulvestrant in advanced breast cancer: evidence to date and place in therapy. Ther Adv Med Oncol. 2017;9(7):465-479.

5. De Placido S, Pronzato $\mathrm{P}$. Treatment options in $\mathrm{HR}^{+} / \mathrm{HER}^{-}$advanced breast cancer patients pretreated with nonsteroidal aromatase inhibitors: what does current evidence tell us? Future Oncol. 2015;11(6):975-981.

6. Deeks ED. Fulvestrant: a review in advanced breast cancer not previously treated with endocrine therapy. Drugs. 2018;78(1):131-137.

7. Robertson JFR, Bondarenko IM, Trishkina E, et al. Fulvestrant 500 $\mathrm{mg}$ versus anastrozole $1 \mathrm{mg}$ for hormone receptor-positive advanced breast cancer (FALCON): an international, randomised, double-blind, phase 3 trial. Lancet. 2016;388(10063):2997-3005.

8. Robertson JF, Llombart-Cussac A, Rolski J, et al. Activity of fulvestrant $500 \mathrm{mg}$ versus anastrozole $1 \mathrm{mg}$ as first-line treatment for advanced breast cancer: results from the FIRST study. J Clin Oncol. 2009;27(27):4530-4535.

9. Johnston SR, Kilburn LS, Ellis P, et al. Fulvestrant plus anastrozole or placebo versus exemestane alone after progression on non-steroidal aromatase inhibitors in postmenopausal patients with hormone-receptorpositive locally advanced or metastatic breast cancer (SoFEA): a composite, multicentre, phase 3 randomised trial. Lancet Oncol. 2013;14(10):989-998.

10. Perey L, Paridaens R, Hawle H, et al. Clinical benefit of fulvestrant in postmenopausal women with advanced breast cancer and primary or acquired resistance to aromatase inhibitors: final results of phase II Swiss Group for Clinical Cancer Research Trial (SAKK 21/00). Ann Oncol. 2007;18(1):64-69.

11. Ciruelos E, Pascual T, Arroyo Vozmediano ML, et al. The therapeutic role of fulvestrant in the management of patients with hormone receptorpositive breast cancer. Breast. 2014;23(3):201-208.

12. Cristofanilli M, Turner NC, Bondarenko I, et al. Fulvestrant plus palbociclib versus fulvestrant plus placebo for treatment of hormonereceptor-positive, HER2-negative metastatic breast cancer that progressed on previous endocrine therapy (PALOMA-3): final analysis of the multicentre, double-blind, phase 3 randomised controlled trial Lancet Oncol. 2016;17(4):425-439.

13. Baselga J, Im SA, Iwata H, et al. Buparlisib plus fulvestrant versus placebo plus fulvestrant in postmenopausal, hormone receptor-positive, HER2negative, advanced breast cancer (BELLE-2): a randomised, doubleblind, placebo-controlled, phase 3 trial. Lancet Oncol. 2017;18(7): 904-916.

14. Sledge GW, Toi M, Neven P, et al. MONARCH 2: abemaciclib in combination with fulvestrant in women with HR+/HER2-advanced breast cancer who had progressed while receiving endocrine therapy. J Clin Oncol. 2017;35(25):2875-2884.

15. Zaman K, Winterhalder R, Mamot C, et al. Fulvestrant with or without selumetinib, a MEK 1/2 inhibitor, in breast cancer progressing after aromatase inhibitor therapy: a multicentre randomised placebo-controlled double-blind phase II trial, SAKK 21/08. Eur J Cancer. 2015 51(10):1212-1220.

16. Burstein HJ, Cirrincione CT, Barry WT, et al. Endocrine therapy with or without inhibition of epidermal growth factor receptor and human epidermal growth factor receptor 2: a randomized, double-blind, placebo-controlled phase III trial of fulvestrant with or without lapatinib for postmenopausal women with hormone receptor-positive advanced breast cancer-CALGB 40302 (Alliance). J Clin Oncol. 2014;32(35):3959-3966.

17. Clemons MJ, Cochrane B, Pond GR, et al. Randomised, phase II, placebo-controlled, trial of fulvestrant plus vandetanib in postmenopausal women with bone only or bone predominant, hormone-receptorpositive metastatic breast cancer (MBC): the OCOG ZAMBONEY study. Breast Cancer Res Treat. 2014;146(1):153-162.

18. Jadad AR, Moore RA, Carroll D, et al. Assessing the quality of reports of randomized clinical trials: is blinding necessary? Control Clin Trials. 1996;17(1):1-12.

19. Xu L, Wu X, Hu C, et al. A meta-analysis of combination therapy versus single-agent therapy in anthracycline- and taxane-pretreated metastatic breast cancer: results from nine randomized Phase III trials. Onco Targets Ther. 2016;9:4061-4074.
20. Zintzaras E, Ioannidis JP. Heterogeneity testing in meta-analysis of genome searches. Genet Epidemiol. 2005;28(2):123-137.

21. Dersimonian R, Laird N. Meta-analysis in clinical trials. Control Clin Trials. 1986;7(3):177-188.

22. Mantel N, Haenszel W. Statistical aspects of the analysis of data from retrospective studies of disease. J Natl Cancer Inst. 1959;22(4): 719-748.

23. Adelson K, Ramaswamy B, Sparano JA, et al. Randomized phase II trial of fulvestrant alone or in combination with bortezomib in hormone receptor-positive metastatic breast cancer resistant to aromatase inhibitors: a New York Cancer Consortium trial. NPJ Breast Cancer. 2016;2:16037

24. Hyams DM, Chan A, de Oliveira C, et al. Cediranib in combination with fulvestrant in hormone-sensitive metastatic breast cancer: a randomized Phase II study. Invest New Drugs. 2013;31(5):1345-1354.

25. Musolino A, Campone M, Neven P, et al. Phase II, randomized, placebo-controlled study of dovitinib in combination with fulvestrant in postmenopausal patients with $\mathrm{HR}^{+}, \mathrm{HER}^{-}$breast cancer that had progressed during or after prior endocrine therapy. Breast Cancer Res. 2017;19(1):18.

26. Kornblum NS, Manola J, Klein P, et al. Abstract S1-02: PrECOG 0102: a randomized, double-blind, phase II trial of fulvestrant plus everolimus or placebo in post-menopausal women with hormone receptor (HR)-positive, HER2-negative metastatic breast cancer (MBC) resistant to aromatase inhibitor (AI) therapy. Cancer Res. 2017;77(4 Supplement):S1-S2.

27. Krop IE, Mayer IA, Ganju V, et al. Pictilisib for oestrogen receptorpositive, aromatase inhibitor-resistant, advanced or metastatic breast cancer (FERGI): a randomised, double-blind, placebo-controlled, phase 2 trial. Lancet Oncol. 2016;17(6):811-821.

28. Di Leo A, Johnston S, Lee KS, et al. Buparlisib plus fulvestrant in postmenopausal women with hormone-receptor-positive, HER2-negative, advanced breast cancer progressing on or after mTOR inhibition (BELLE-3): a randomised, double-blind, placebo-controlled, phase 3 trial. Lancet Oncol. 2018;19(1):87-100.

29. Robertson JF, Ferrero JM, Bourgeois H, et al. Ganitumab with either exemestane or fulvestrant for postmenopausal women with advanced, hormone-receptor-positive breast cancer: a randomised, controlled, double-blind, phase 2 trial. Lancet Oncol. 2013;14(3):228-235.

30. Robertson JF, Osborne CK, Howell A, et al. Fulvestrant versus anastrozole for the treatment of advanced breast carcinoma in postmenopausal women: a prospective combined analysis of two multicenter trials. Cancer. 2003;98(2):229-238.

31. Howell A, Pippen J, Elledge RM, et al. Fulvestrant versus anastrozole for the treatment of advanced breast carcinoma: a prospectively planned combined survival analysis of two multicenter trials. Cancer. 2005;104(2):236-239.

32. Lin WZ, Xu QN, Wang HB, Li XY, Qn X, Xy L. Fulvestrant plus targeted agents versus fulvestrant alone for treatment of hormone-receptor positive advanced breast cancer progressed on previous endocrine therapy: a meta-analysis of randomized controlled trials. Breast Cancer. 2017;24(3):345-352.

33. Wang R, Li X, Zhang H, Wang K, He J. Cell-free circulating tumor DNA analysis for breast cancer and its clinical utilization as a biomarker. Oncotarget. 2017;8(43):75742-75755.

34. Reinhardt F, Franken A, Fehm T, Neubauer H. Navigation through interand intratumoral heterogeneity of endocrine resistance mechanisms in breast cancer: a potential role for Liquid Biopsies? Tumour Biol. 2017; 39(11):101042831773151.

35. Liu CY, Wu CY, Petrossian K, Huang TT, Tseng LM, Chen S. Treatment for the endocrine resistant breast cancer: current options and future perspectives. J Steroid Biochem Mol Biol. 2017;172:166-175.

36. Tryfonidis K, Zardavas D, Katzenellenbogen BS, Piccart M. Endocrine treatment in breast cancer: cure, resistance and beyond. Cancer Treat Rev. 2016;50:68-81.

37. Zhang P, Li CZ, Jiao GM, et al. Effects of ovarian ablation or suppression in premenopausal breast cancer: a meta-analysis of randomized controlled trials. Eur J Surg Oncol. 2017;43(7):1161-1172. 
38. Schmid P, Untch M, Kossé V, et al. Leuprorelin acetate every-3-months depot versus cyclophosphamide, methotrexate, and fluorouracil as adjuvant treatment in premenopausal patients with node-positive breast cancer: the TABLE study. J Clin Oncol. 2007;25(18):2509-2515.
39. Di Leo A, Jerusalem G, Petruzelka L, et al. Results of the CONFIRM phase III trial comparing fulvestrant $250 \mathrm{mg}$ with fulvestrant $500 \mathrm{mg}$ in postmenopausal women with estrogen receptor-positive advanced breast cancer. J Clin Oncol. 2010;28(30):4594-4600.

\section{Publish your work in this journal}

OncoTargets and Therapy is an international, peer-reviewed, open access journal focusing on the pathological basis of all cancers, potential targets for therapy and treatment protocols employed to improve the management of cancer patients. The journal also focuses on the impact of management programs and new therapeutic agents and protocols on

\section{Dovepress}

patient perspectives such as quality of life, adherence and satisfaction The manuscript management system is completely online and includes a very quick and fair peer-review system, which is all easy to use. Visit http://www.dovepress.com/testimonials.php to read real quotes from published authors.

Submit your manuscript here: http://www.dovepress.com/oncotargets-and-therapy-journal 